\title{
Conducting Low Cost Community-Engaged Health Research with Deaf American Sign Language Users
}

\author{
Tyler G. James \\ University of Florida \\ Michael M. McKee \\ University of Michigan \\ Glenna R. Ashton \\ Deaf Community Advisory Workgroup \\ David G. Phillips
}

\author{
Meagan K. Sullivan \\ University of Florida \\ Stephen J. Hardy \\ Deaf Community Advisory Workgroup \\ Zulma Santiago Zayas \\ Deaf Community Advisory Workgroup \\ JeeWon Cheong \\ University of Florida
}

\begin{abstract}
Background. Deaf American Sign Language (ASL) users represent an understudied linguistic/cultural minority group experiencing social disenfranchisement leading to a myriad of health inequities. Community-engaged and community-based participatory research is a best practice when working with Deaf ASL-users; yet, prior research using large funding mechanisms from governmental organizations is mostly unattainable by community organizations. The purpose of this study was to conduct a pilot survey assessing the feasibility of community-engagement methods to administer a low cost, community-oriented, accessible health survey among Deaf ASL-users in Florida. Methods. We established a community advisory workgroup of representatives from major Deaf-serving organizations and higher density localities. The community-academic partnership developed an accessible health survey available in ASL and English. The pilot survey was administered over a three-month period in summer 2018 using convenience sampling strategies including in-person recruitment at community events, and advertisements through Facebook and community e-mail lists. Results. Our recruitment strategy yielded 92 eligible, and diverse, respondents from across the state of Florida. We provide lessons learned from our strategy including considerations for including community members as translation reviewers; recruiting participants across a large geographic area with limited time; and, informed consent strategies. Conclusions. Limited resource environments are pervasive in public health practice and in the communities we serve. Using data from this pilot survey, we identified recommendations for future study methodology to improve community-orientation and participant recruitment among this priority population.
\end{abstract}

Supplementary material and other study-related information: $\underline{\text { https://osf.io/qu489/ }}$

Tyler G. James, Meagan K. Sullivan, and JeeWon Cheong, Department of Health Education and Behavior, University of Florida; Michael M. McKee, Department of Family Medicine, University of Michigan; Stephen J. Hardy, Deaf Community Advisory Workgroup and Department of Speech, Language, and Hearing Sciences, University of Florida; Glenna R. Ashton, Deaf Community Advisory Workgroup; Zulma Santiago Zayas, Deaf Community Advisory Workgroup; David G. Phillips, unaffiliated.

Portions of this paper were presented as a poster at the 2018 Society for Public Health Education Annual Meeting in Columbus, Ohio and to the Disability Community Planning Group of the Florida Disability and Health Program/Florida Department of Health. There is no funding to report for this paper.

The authors wish to thank Danielle R. Dorn and Ashleigh T. Poole for assisting with geographic data compilation and analysis. We are grateful to David Maruca for serving as an interpreter for this project, and to Kelley Wyse at the University of Michigan for reviewing our survey translations. We also thank the Florida Association for the Deaf, the Florida Disability and Health Program, regional associations for the Deaf, and the broader Florida Deaf community for supporting this research. Correspondence concerning this paper should be addressed to Tyler G. James (tjames95@ufl.edu). 
Community-engaged/community-based participatory research (CEnR and CBPR) are becoming public health best practice due to improved understanding of the contextual factors within the research or programmatic environment, enhanced recruitment of hard-toreach populations, translation of research findings to practice, empowerment of disadvantaged communities, and superior dissemination opportunities (Israel et al., 2005, 2010; Minkler, 2005). These benefits are gained by giving community members an active role in the development and implementation of the research project. This allows community members to be engaged throughout the research process with academic researchers. However, the value added to health research using CEnR/CBPR approaches is juxtaposed to the well-established challenges including resource complexity - for community members, researchers, and community organizations -, time intensiveness, barriers to ensuring consistent community participation, communication and relationship demands, and a multitude of ethical challenges for researchers and academic institutions (Israel et al., 2005; Mikesell et al., 2013; Minkler, 2005). Due to these barriers and additional formative challenges of CBPR/CEnR, researchers typically withhold from community-engaged projects until large-scale funding mechanisms are available to better leverage institutional and community resources, facilitate participation, and ensure program sustainability (Logan et al., 2010; Simonds et al., 2013).

An example where this has become evident is within the field of $\mathrm{d} /$ Deaf and hard-of-hearing (hereinafter: DHH) health equity research. Over the past two decades research has highlighted the benefits of CEnR/CBPR-methods in health disparities research among DHH American Sign Language (ASL)-users, who are understudied - often attributed to linguistic and cultural challenges to recruitment in health surveillance research (S. L. Barnett et al., 2011). These studies have highlighted that $\mathrm{DHH}$ individuals often experience health disparities ranging from higher emergency department utilization (S. L. Barnett et al., 2017; McKee et al., 2015), lower health literacy (Kushalnagar, Ryan, et al., 2017; McKee et al., 2015), and poorer patient-provider communication (Kushalnagar et al., 2018; McKee et al., 2011) due to widespread harmful social determinants of health, likely partly attributable to language deprivation (Hall, 2017; Hall et al., 2017). The majority of this research employed CEnR/CBPR methods in areas with larger per capita $\mathrm{DHH}$ populations while being funded by cooperative agreements and research grants from the Centers for Disease Control and Prevention (CDC) and the National Institutes of Health (NIH). For example, the CDC-funded Prevention Research Center in Rochester, NY, National Center for Deaf Health Research (NCDHR), has been recognized as establishing a precedent for CBPR partnerships within the CDC Prevention Resource Network by engaging the Rochester DHH community in research collaboration (Hawkins-Cox et al., 2014). This allowed them to adapt and translate previously inaccessible surveys and promote recruitment into research projects. Other research groups have leveraged connections with community organizations and service centers, providing similar benefits (Kushalnagar, Harris, et al., 2017). However, the resource intensiveness of community-engaged work is further emphasized: research programs are funded by NIH research mechanisms unlikely to be funded without proof of concept and community capacity, and scientific novelty - while NCDHR is funded by the CDC's Prevention Research Centers program and can leverage this resource and their academic-community partnership to apply for additional funding (Hawkins-Cox et al., 2014).

Applying CEnR/CBPR methods is important to promote social justice in health research with historically disadvantaged populations such as the DHH community. Yet, exemplar cases do not facilitate application to different contexts, particularly in lower resource settings experienced by many DHH communities and organizations across the country. The costs of CEnR/CBPR often pose a significant barrier to empowering communities to activate change within their localities and present roadblocks for organizations and researchers interested in community-engaged projects.

Pilot studies can help alleviate these challenges by better understanding resource needs and providing information about methodological feasibility within a particular setting. Pilots are focused on identifying methods to improve study design for future studies and can be beneficial for early career investigators to gain experience with collaborating, writing grants, and working with stakeholders (Moore et al., 2011). However, integrating pilot studies within a CEnR/CBPR approach can be difficult if investigators do not yet have productive partnerships within the community of interest (Wethington et al., 2007).

The current study employed CEnR methods to conduct a pilot survey among DHH ASL-users from Florida to assess methodological feasibility and recruitment strategies when working with limited financial re- 
sources (i.e., \$1,500 available). We aimed to (1) understand optimal levels of community-based engagement and (2) test our low-cost recruitment methods to develop recommendations for future $\mathrm{DHH}$ communitybased studies.

\section{Methods}

\section{Community Engagement}

This study was conceived through conversations with the DHH ASL-using community in Florida starting in 2016. DHH community leaders throughout the state (e.g., the Florida Association of the Deaf [FAD]) recognized the need to have more empirical data to validate healthcare access issues and chronic health conditions they observed in their communities; yet, there had been no formal investigation focusing on the $\mathrm{DHH}$ community's health in Florida. The study's lead investigator (James) had several meetings with mentors (McKee, Cheong) and community members in the local region (e.g., Hardy) to discuss developing a survey similar to the Rochester Deaf Health Survey administered by NCHDR but focusing on the needs of DHH ASLusers living in Florida. Given James's background and experience in the Florida DHH ASL-using community, we (James, McKee, Cheong) decided to implement CEnR techniques to conduct a pilot survey and Hardy was invited to serve as a member of the Deaf Community Advisory Workgroup (DCAW). We agreed to be purposeful in selecting members of the DCAW based on geographic location and community-based organization (CBO) affiliation. For example, Hardy was located in north central Florida, was the former president of FAD and has served multiple terms as a governorappointed member of the Florida Vocational Rehabilitation Council. As DCAW members would be encouraged to assist with recruitment, it was vital they have well established networks within the Florida DHH ASL community.

Prior to selecting DCAW members, we requested data from the Florida Division of Vocational Rehabilitation (VR) to understand the geographic distribution of DHH customers by county. VR is a division of each state's department of education that provides job skills training and services to individuals with disabilities to reduce unemployment. These data only represent individuals seeking VR's services; however, given the fact that DHH Floridians are significantly un/underemployed (Florida Coordinating Council for the Deaf and Hard of Hearing, 2017; Garberoglio et al., 2017), VR data provides a glimpse at the location of larger $\mathrm{DHH}$ communities. Concurrently, we conducted an online search and developed a list of DHH serving organizations and religious institutions in Florida that would later be contacted to assist in recruitment. We used free, open-source packages in the $\mathrm{R}$ statistical environment (i.e., choroplethr) (Lamstein, 2018) to develop geographic information system (GIS) products with county frequency and organization data. These maps were later revised using the university's license of ArcGIS (see Figure 1). Larger concentrations of DHH VR customers were observed in south Florida (near Miami-Dade County) and Pinellas and Hillsborough counties (Tampa and Clearwater).

This map guided James's and Hardy's discussions of prospective DCAW members leading to the inclusion of Ashton - governor-appointed representative on the Florida Coordinating Council for the Deaf and Hard-of-Hearing and former secretary of FAD - from South Florida, and Santiago Zayas - from the Tampaarea, who is heavily involved in the Puerto Rican Deaf community in Florida and who volunteered at a large, DHH-focused CBO in Pinellas county. Early attempts to invite other members from underrepresented cultural and geographic communities were unsuccessful due to their personal illness, and organizational restructuring and dissolution.

We requested the three-member DCAW be as involved as they preferred, but at minimum provide consultation and collaboration regarding the development of the survey, recruitment and data collection strategies, and guidance with reporting and disseminating findings. In addition, they connected us with $\mathrm{DHH}-$ serving CBOs who could help with recruitment and dissemination.

Due to differing schedules of DCAW members, and to be respectful of their volunteered time, James met via videoconference individually to discuss study progress and receive feedback. Updates from these meetings were sent to the DCAW in group e-mails with fulllength updates in ASL; DCAW members were encouraged to discuss and share feedback with the academic team. After data collection completed (i.e., September 2018), we met as a group bi-monthly, as needed, via videoconference to discuss project updates, results, and next steps.

\section{Survey Development}

The academic team presented the DCAW a preliminary table of content specifications based on prior health disparities research focused on DHH ASL-users. The DCAW prioritized surveying mental health, health 


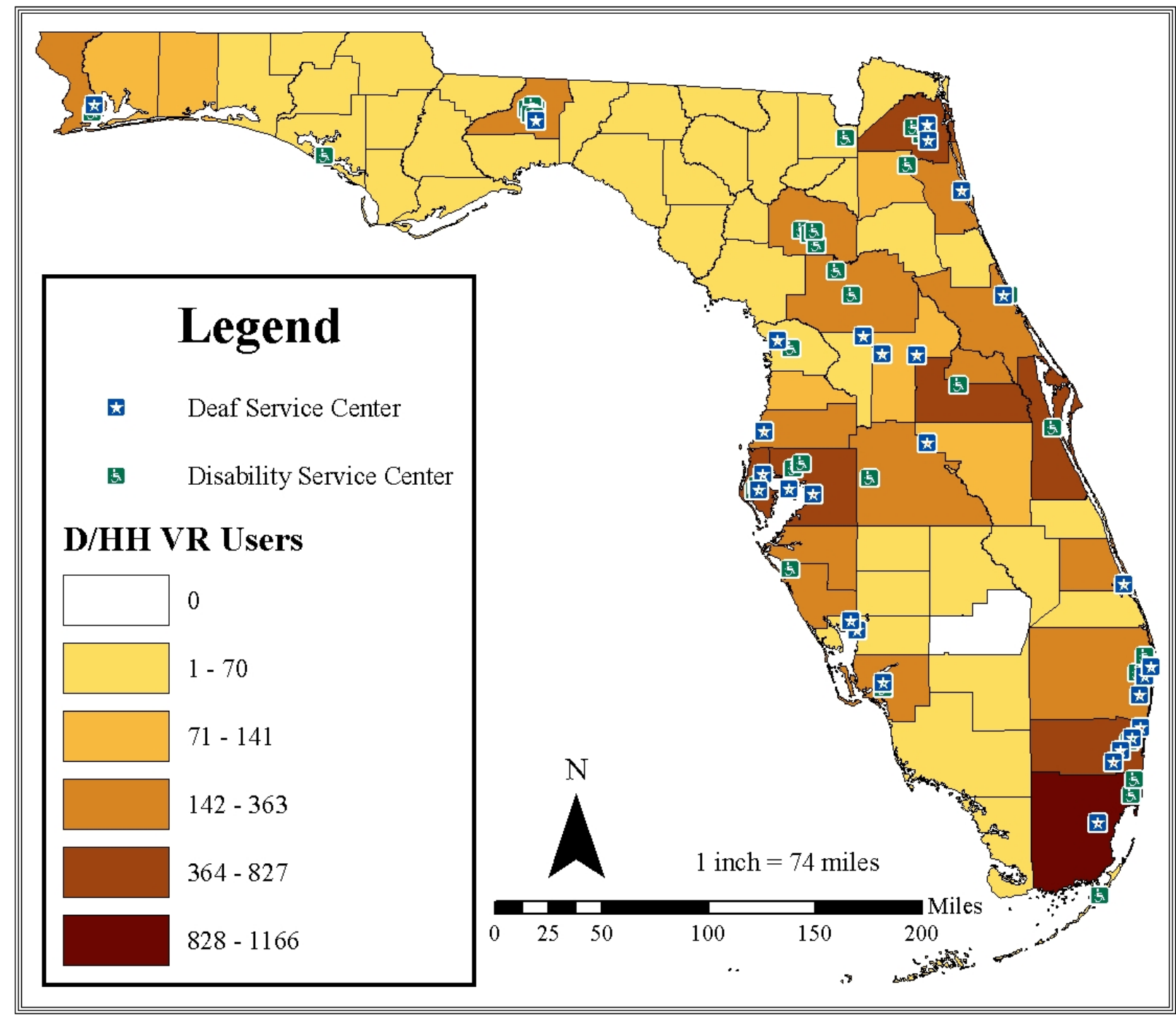

Figure 1. Distribution of (1) deaf and hard-of-hearing vocational rehabilitation (VR) customers, between January 1, 2012 and June 27, 2017, (2) deaf and hard-of-hearing service centers or organizations (including memberbased community organizations), and (3) disability service centers. VR data is not exclusive to DHH ASL-users.

needs of community members and obtaining interpreter services for healthcare encounters. After several iterations of item revision (via e-mail and in-person discussion), we created the final survey. Most questionnaire items were benchmarked from previous studies with DHH individuals (S. Barnett et al., 2011), in addition to the ASL-version of the Newest Vital Signs (McKee et al., 2015) - a health literacy measure -, the Patient Health Questionnaire-2 (a depression screener) (Kroenke et al., 2003) originally translated into ASL by the University of Michigan's Department of Family Medicine (Pertz et al., 2018), and national health surveys (i.e., CDC's Behavioral Risk Factor Surveillance System and the National Health Interview Survey). See
Supplemental File 1 (https://osf.io/qu489/) for full survey in English.

Translation. First, we submitted the English survey for preliminary IRB approval (e.g., survey items and methods were suitable for the type of review selected) before proceeding with the translation process. After receiving approval contingent on translation verification, James worked closely with a Deaf ASL-user who has extensive interpreting and translation experience - to translate the written questionnaire items into ASL. This "first draft" translation was guided by translation principles of previous ASL health surveys (i.e., seeking comprehension for the greatest number of respondents, conceptual equivalence, and addressing fund of information gaps) (Graybill et al., 2010), with 
feedback from James to ensure fidelity to the health construct.

Translation drafts were revised prior to DCAW review. DCAW members were requested to provide individual feedback using a rubric measuring each item's conceptual accuracy of the ASL item wording and response options in comparison to the written English item (all DCAW members are bilingual in ASL and English), and subjective determination of translation clarity for a variety of DHH ASL-users. TGJ reviewed all rubrics, discussed conflicting responses with DCAW members, and requested revisions.

The final phase of the survey translation process was a blind back-translation by Phillips, who only had access to the general purpose of the study and the ASL translated survey. Back-translation is a process of translating from the target language (in this case, ASL) back into the source language (English) to ensure translation accuracy (Brislin, 1970). Phillips, a nationally certified hearing interpreter with degrees in linguistics and community interpreting, back-translated each survey question from ASL to written English. Both Phillips and the DCAW were empowered as the final, study-level decision-makers for translation accuracy; in cases where revisions were necessary, Phillips worked with the original translator and the DCAW to determine a linguistically and culturally appropriate translation.

\section{Informed Consent}

Federal regulations (i.e., 45 CFR $\S 46.116$ ) require that researchers provide informed consent documents in the language most understood by the participant; typically, this is the participant's preferred language. Due to limited English proficiency (i.e., average English reading level of DHH ASL-using adults similar to a 6th grader) (Zazove et al., 2013) and "fund of information deficits" (Pollard, 1998) caused by language deprivation and reduced opportunities to incidental learning (Hall et al., 2017), standard informed consent processes that rely on written English forms may be ineffective for DHH ASL-users (McKee et al., 2013). Accordingly, researchers at NCDHR and other centers have developed recommendations for accessible informed consents. These recommendations suggest that informed consents should ideally be provided with language concordant, dialogic, short videos that do not rely on prose, with an "easy-to-read" English consent or be consented by ASL-fluent and culturally competent research staff in person (McKee et al., 2012, 2013). Importantly, other than the aforementioned guidance, there is not yet evidence defining best practices for informed consent of DHH ASL-users; research, funded by the National Institute of Deafness and Other Communication Disorders, is underway at the University of Rochester Medical Center to evaluate differences in effectiveness of informed consent modalities

(5UL1TR000042-10). Therefore, we closely followed McKee and colleague's $(2012,2013)$ guidelines.

After integrating initial feedback from the DCAW on sentence structure and word choice, we submitted the English informed consent for IRB approval; then, James worked with Hardy and the Deaf interpreter who performed as actors (a prospective participant and research team member, respectively) in the informed consent video. Recording took place in the university's free studio over two 8-hour days in December 2017. Phillips oversaw the translation process and provided feedback on target language semantic choices. During the recording process, several recordings 'takes' were made for each section of the informed consent; this provided an opportunity for informed consent actors to provide a baseline translation, receive feedback regarding required content and structure from James and Phillips, and therefore improve the translation quality. At times, actors deviated from the script and the English-based consent document to provide relevant expansion; these changes were decided day-of-recording based on necessity indicated from both actors (one of whom represented the DCAW) and Phillips. The English consent form was subsequently revised to include these expansions, and the other members of the DCAW were informed of these changes.

Phillips back-translated the video into spoken English: providing the ability to easily compare the English document to the video and add closed captions to the ASL video using YouTube ${ }^{\circledR}$ (see Figure 2). DCAW members reviewed the video prior to the study launch to identify any serious mistranslations prior to attesting to the validity of the translated document.

\section{Recruitment}

Our primary recruitment strategy was through snowball sampling through social media (e.g., Facebook $($ ) by posting English text and ASL videos of local DHH community members explaining the purpose of the survey; all video advertisements were less than 1 '30".

We also shared messages through DHH-related CBOs. Specifically, we used the aforementioned list of DHH organizations to disseminate a "Community Partner Recruitment Toolkit." 


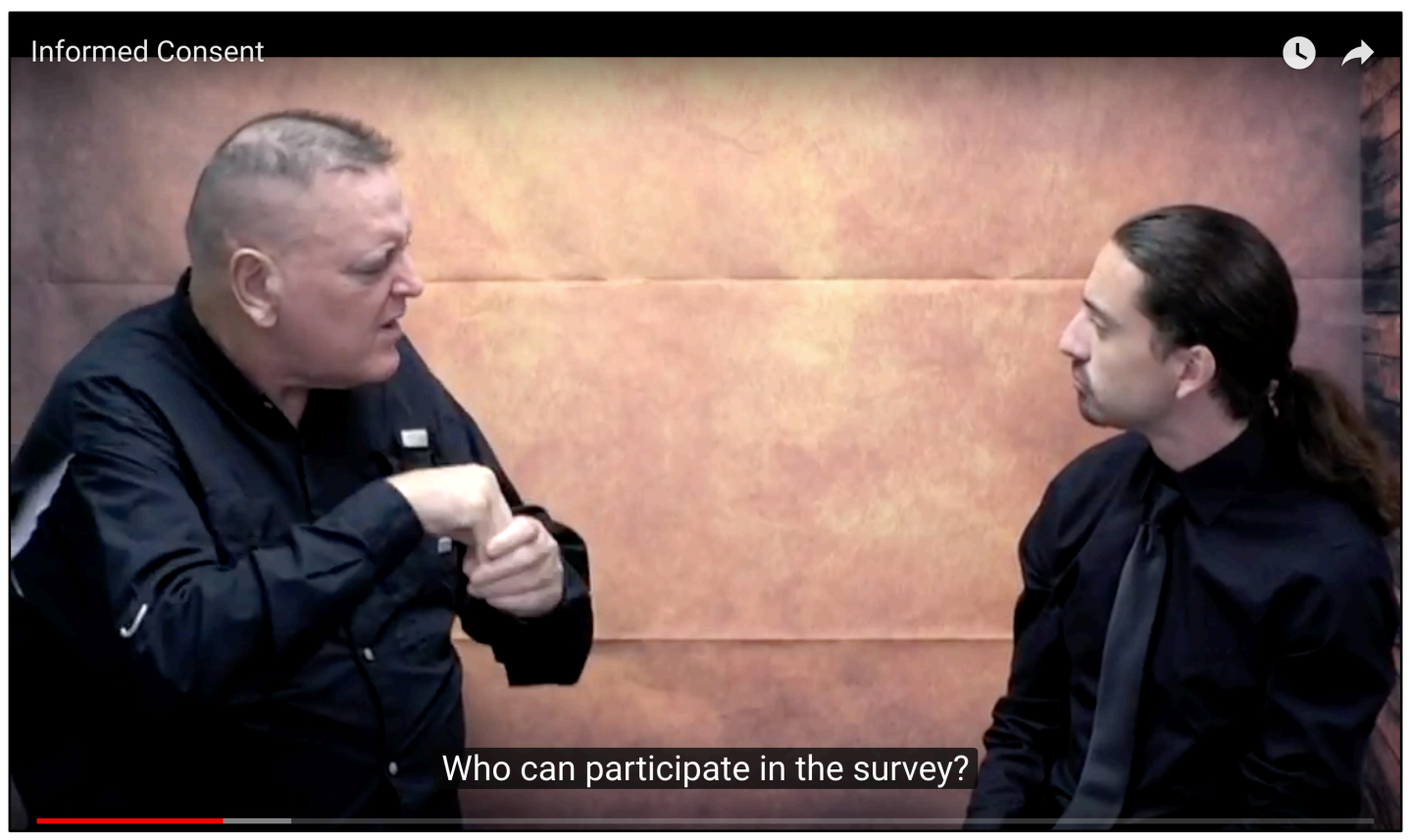

Figure 2. Screenshot of informed consent video showing ASL with English captions.

This toolkit, written in English, described the purpose of the study, provided information on regulatory oversight, introduced the DCAW and research team, acknowledged supporting organizations (e.g., FAD, and Florida Disability and Health Program), and provided guidance on how to use the toolkit (i.e., e-mail templates and social media posts in English and ASL, printable tear-off flyers, and quarter-sheet flyers to disseminate at their discretion). We e-mailed all identified CBOs this document and directly contacted (through videophone) organization leaders in geographically dense areas to discuss the study and the toolkit in more depth. During these discussions, three organization leaders requested printed toolkits and flyers which were subsequently delivered. Lastly, in-person recruitment occurred at DHH community events when a member of the DCAW or the research team could be in attendance. In these situations, we provided prospective participants a quarter-sheet flyer with information on how to access the survey.

\section{Survey Screening and Process}

Advertisements described a screening process to ensure eligibility. Participants were directed to a website to learn more about the study and provided instructions on how to contact the research team for screening. Those who contacted the research team were provided additional information about the survey's risks and benefits, and an opportunity to ask questions about the study.
Eligibility criteria to take the survey was: (1) self-identifying as Deaf, hard-of-hearing, DeafBlind, hearing impaired, or late-deafened; (2) being 18 years or older; (3) living in the state of Florida; and (4) using ASL as a primary form of communication. Interested participants were asked to provide proof of Florida residency and confirm they were over 18 years old. If they met the eligibility criteria, they were provided the survey's generic password and web address. At onset of data collection, the survey did not have appropriate branching logic for the screening and did not have the password protection; this error was realized on the first day of data collection. Thus, to maintain integrity of the survey data and ensure only eligible participants took the survey, we deleted prior responses and ensured the correct, aforementioned screening process was implemented.

The anonymous survey was administered through Qualtrics. Participants were provided the informed consent and, upon agreeing, accessed the survey. The first three questions of the survey were screening confirmation items; failure to answer within the inclusion criteria automatically forced-exit the survey. At the end of the survey, participants were provided the opportunity to complete an unlinked incentive form, to receive a $\$ 10$ Amazon ${ }^{\circledR}$ or Visa gift card. 


\section{Results}

\section{Survey Translation}

The start of survey translation was intended for August 2017; however, this was delayed until mid-September due to damage from Hurricane Irma. DCAW completed translation review on October 10, 2017. Two DCAW members reviewed the translations; percent agreement of translation accuracy was high for question translations (92\%) and clarity of sign choice and syntax (89\%), while raters agreed on response option translation accuracy $78 \%$ of the time. A total of 13 items were revised prior to blind back-translation; these items were predominately demographic in nature (e.g., DHH-related identities, race/ethnicity, education status), with revisions necessary to ensure that sign choices were culturally appropriate and understood by a variety of respondents. We experienced similar translation challenges highlighted by Graybill and colleagues due to linguistic variance between ASL and English (e.g., the word "drink" has multiple sign variations in ASL) (Graybill et al., 2010). For example, there was substantial discussion on the amount of expansion necessary to elicit sexual health concerns: in ASL, sign choices differ depending on the specific behavior. Based on DCAW recommendations, we used expansion to include reproductive and sexual health (i.e., birth control, condom use, STD risk). In addition, we incorporated imagery and expansion where necessary (e.g., for the binge drinking item). Blind back-translation began and finished in October 2017. All items and response options had linguistic equivalence to the original content and, thus, were appropriate for final recording.

\section{Recruitment}

The study period was from June 2018 to September 2018. Our social media recruitment videos were shared a total of 118 times and had approximately 4,100 views. Figure 3 provides recruitment activities and participation by study week. Of note, during study week 11, an unaffiliated community member distributed their own video encouraging community participation. This video was shared 26 times and received $\sim 1,500$ views.

Our recruitment strategy yielded 92 eligible respondents diverse in age (18 to 80 years), education status, income, and DHH-related demographics (Table 1). Five individuals were deemed ineligible (four were DHH English-speakers from Florida, and one was a
DHH ASL-user from Pennsylvania). Women, white individuals, and those with college education were overrepresented in our sample. We reduced the resolution of residential data to ensure protection of research participants by using U.S. Census Defined Statistical Areas instead of county-level data. Our sample included individuals across the state of Florida with more participants in locations of more DHH VR customers and, thus, where our DCAW members lived.

\section{Informed Consent}

The English informed consent was estimated by online software to be between read in approximately 4'30" and 6' 10"; the ASL video view time was 11'50". According to YouTube, there were only 76 views of the informed consent video; in contrast, there were 92 individuals who started the survey. The average consent video view duration of participants who consented was 5'16". Importantly, information on risks and protections occurred prior to 4'14'". The average total time on the informed consent webpage was 4'7' ( $\mathrm{SD}=5$ '56", med. = 1'3", range: 4'38' to 19'46"). Only one participant contacted the research team prior to agreeing to the informed consent to learn about risks and benefits.

\section{Cost Estimation}

In total, we spent approximately 1,220 USD not including salary of academic-based research team members; total effort estimates of faculty members and graduate students equal approximately 200 hours. The majority (\$820) of financial resources were used to cover the cost of recruitment materials and incentives. Interpreter compensation was negotiated through clear communication on financial resources, and personal and professional goals (Phillips compensated through authorship on scholarly outputs, a priority for his career; and the Deaf translator/actor was paid $\$ 400$ and rights to use materials for his interpreting portfolio). An open conversation occurred between DCAW members and James regarding financial resources and capacity building for future projects, and all DCAW members agreed that this project's implications for their community was more important than financial compensation. In the end, DCAW members described their experience positively: they felt that the research team was attentive to feedback and concerns and empowered the DCAW to make decisions about the research process. Based on the level of work provided throughout the project, all DCAW members were provided co-authorship on scholarly outputs. 


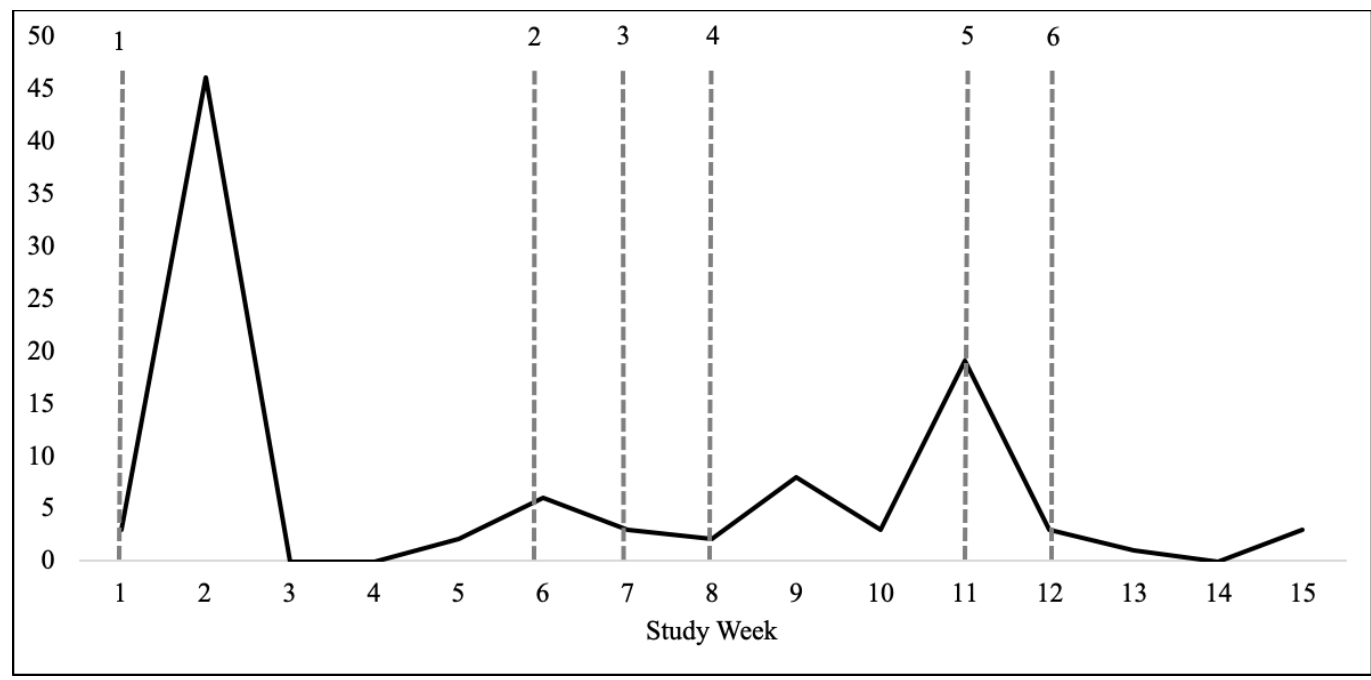

Figure 3. Number of participants joining the study per week with major project recruitment phases. Recruitment milestones include: (1) Initial advertisement; (2) E-mail from the Florida Association of the Deaf and in-person recruitment; (3) Advertisement by a regional organization in West Florida; (4) In-person recruitment at multiple central Florida events; (5) Unaffiliated Deaf community member sharing video; and (6) In-person recruitment in north central Florida.

Table 1

Sample characteristics of the 2018 pilot survey sample.

\begin{tabular}{l|c}
\hline \multicolumn{1}{c|}{ Characteristic } & $\mathbf{N = 9 2}$ \\
\hline Age, Mean (SD) & 43.2 years (15.0) \\
Gender & Range: 18 to 80 \\
Female & $65.2 \%(60)$ \\
Male & $34.8 \%(32)$ \\
Race $\quad$ White & $78.3 \%(72)$ \\
African American/Black & $6.5 \%(6)$ \\
Asian & $0.0 \%(0)$ \\
Pacific Islander & $0.0 \%(0)$ \\
American Indian or Alaskan Native & $1.1 \%(1)$ \\
Biracial/Multiracial/Other & $14.1 \%(13)$ \\
Hispanic/Latino & $25.0 \%(23)$ \\
Education & $4.3 \%(4)$ \\
Less than high school diploma or GED & $16.3 \%(15)$ \\
High school graduate & $35.9 \%(33)$ \\
Some college or technical school & $43.5 \%(40)$ \\
College graduate & $56.2 \%(51)$ \\
Employed & \\
Household income & \\
$<\$ 15 \mathrm{k}$ & $20.5 \%(17)$ \\
\$15 $-\$ 25 \mathrm{k}$ & $22.9 \%(19)$ \\
\$25k $-\$ 50 \mathrm{k}$ & $28.9 \%(24)$ \\
$>$ \$50k & $27.7 \%(23)$ \\
Age became DHH & \\
Born deaf & $51.6 \%(47)$ \\
\end{tabular}


Deaf before or at age 3

$33.0 \%(30)$

Deaf after age 3

$15.4 \%(14)$

Deaf education history ${ }^{\mathrm{a}}$

Did not attend school

Attended a school for the Deaf

Did not attend a school for the Deaf

Attended both a school for the Deaf and mainstream school

Geographic-area (i.e., Census Defined Statistical Area)

Miami-Fort Lauderdale-Port St. Lucie CSA

Orlando-Deltona-Daytona Beach CSA

Jacksonville-Palatka-St. Marys CSA

Sarasota-Bradenton-Punta Gorda CSA

Not assigned CSA

Tampa-St. Petersburg-Clearwater, FL MSA

Cape Coral-Fort Myers, FL MSA

Lakeland-Winter Haven, FL MSA

Palm Bay-Melbourne-Titusville, FL MSA

Pensacola-Ferry Pass-Brent, FL MSA

Tallahassee, FL MSA

Ocala, FL MSA

Naples-Macro Island, FL MSA

Gainesville, FL MSA

Fort Walton Beach-Crestview-Destin, FL MSA

Panama City-Lynn Haven, FL MSA

Homosassa Springs, FL $\mu$ SA

Sebring, FL $\mu$ SA

Key West, FL $\mu$ SA

Lake City, FL $\mu$ SA

Clewiston, FL $\mu$ SA

Arcadia, FL $\mu$ SA

Wauchula, FL $\mu$ SA

$4.3 \%(4)$

$16.3 \%(15)$

$35.9 \%(33)$

$43.5 \%(40)$

$17.4 \%(16)$

$15.2 \%(14)$

$5.4 \%(5)$

$1.1 \%(1)$

$57.6 \%(53)$

30

0

3

1

2

1

0

1

10

2

0

1

0

0

2

0

0

0

Not assigned MSA or $\mu$ SA

$3.3 \%(3)$

${ }^{a}$ Data missing on some cases.

$\mathrm{CSA}=$ Core Statistical Area MSA $=$ Metropolitan Statistical Area $\mu \mathrm{SA}=$ Micropolitan Statistical Area

\section{Discussion}

DHH ASL-users are an understudied population and, to date, most health research studying this population is conducted using large funding mechanisms from national organizations. This limits the application of methods to smaller localities, not funded by research mechanisms. Therefore, the purpose of this study was to conduct a pilot feasibility study to understand the application of CEnR methods at the state-level, at lowcost. Specifically, our approach used methods alternative to those presented in the literature for: translation, recruitment, and informed consent.

Our translation methods presented both strengths and challenges. The online review method allowed community members to assess translations when and where most convenient. However, it is possible that in- person review may have provided faster and richer feedback. Future studies incorporating this type of translation review method should allow flexibility in their timelines for unanticipated circumstances (e.g., extreme weather events).

There are several lessons learned from our recruitment process. First, using social media as a primary recruitment mechanism was beneficial because there are large groups on these platforms of DHH community members bounded by a geographic area; this provided a low-cost method of disseminating our survey. In retrospect, it may be more beneficial to engage wellknown community members from each region to film advertisement videos. This may help increase recruitment of people in harder to reach areas (e.g., rural areas). Further, our focus on social media and in-person recruitment appeared to introduce sampling bias: $\mathrm{DHH}$ 
individuals from disadvantaged backgrounds may not have the resources necessary to have been exposed to our messages (e.g., access to a computer, access to attend community events). Secondly, our Community Partner Recruitment Toolkit was only available in English and was not published on the study's website, limiting access to other organizations and Deaf individuals who prefer receiving information in ASL. Future studies should ensure that all study information is available in both the predominant language and preferred language of the community.

Additional research is needed on effective recruitment methods for DHH ASL-users. In 2013, McKee and colleagues acknowledged the potential to use Respondent Driven Sampling (RDS) methods in DHH health research (McKee et al., 2013). RDS is a chainreferral method of sampling that allows researchers to gain access to hard-to-reach populations, while providing a statistical correction to estimate population-level statistics (Heckathorn, 2002). We echo McKee and colleagues' recommendation as our CEnR approach, including the community member who self-published a video advertisement, suggests these methods may be appropriate for this population. However, there is a need to assess the methodological implementation of RDS in Deaf health research. Further, additional research is necessary on how to develop appropriate, noncoercive incentives for this population; several eligible participants commented on social media, in-person, and at time of screening that the incentive was not enough and refused to participate. Similarly, future projects should work to ensure compensation for $\mathrm{DHH}$ community partners who are providing their valuable time and expertise to expanding DHH health research. This compensation is in addition to adhering to the necessary reciprocity tenant of CEnR/CBPR research and building community capacity.

Finally, our informed consent findings highlight the need to identify effective strategies for obtaining informed consent from DHH participants. We note that the time spent on the informed consent form (both ASL and English) does not necessarily equate to comprehension of the informed consent. In keeping with low cost methods, our informed consent methodology included a dialogic video with two actors portraying potential questions and responses about the study. Studies with more resources may consider using a live video-messaging platform to consent participants - providing more rich information dialogue, allowing participants to ask questions about the study and permitting researchers to check for comprehension of study materials.

\section{Conclusion}

Limited resource environments are quite common in public health practice and within the communities that we serve but can hinder best practice communityengagement principles that enhance the research process. The purpose of this pilot study was to assess the potential of CEnR methods to conduct health-related surveys with DHH populations with the added challenge of limited financial resources. This information can be used by CBOs and early career researchers to identify strategies to conduct community health assessments and similar projects while maintaining community-engagement. We identified several opportunities for improving future study methodology including potentially effective recruitment and informed consent strategies and stronger collaboration opportunities with CBOs. Research products comparing results of the survey to the Florida BRFSS results (consisting of DHH and hearing English-speakers) are currently in progress. In addition to research products, and in practicing reciprocity, we have created advocacy tools for the DHH community in Florida to advocate for additional resources. Lastly, due to the increased capacity for research and collaboration, we successfully obtained a small grant to conduct an additional study on healthcare communication with this population.

\section{References}

Barnett, S., Klein, J. D., Pollard, R. Q., Jr., Samar, V., Schlehofer, D., Starr, M., Sutter, E., Yang, H., \& Pearson, T. A. (2011). Community participatory research with Deaf sign language users to identify health inequities. American Journal of Public Health, 101(12), 2235-2238.

Barnett, S. L., Matthews, K. A., Sutter, E. J., DeWindt, L. A., Pransky, J. A., O’Hearn, A. M., David, T. M., Pollard, R. Q., Jr., Samar, V. J., \& Pearson, T. A. (2017). Collaboration with Deaf communities to conduct accessible health surveillance. American Journal of Preventive Medicine, 52(3), S250-S254. https://doi.org/10.1016/j.amepre.2016.10.011

Barnett, S. L., McKee, M., Smith, S. R., \& Pearson, T. A. (2011). Deaf sign language users, health inequities, and public health: Opportunity for social justice. Preventing Chronic Disease, 8(2). http://www.ncbi.nlm.nih.gov/pmc/articles/PMC3073438/

Brislin, R. W. (1970). Back-translation for cross-cultural research. Journal of Cross-Cultural Psychology, 1(3), 185-216.

Florida Coordinating Council for the Deaf and Hard of Hearing. (2017). 2017 FCCDHH Biennial Report to Governor Rick Scott, the Florida Legislature \& the Supreme Court. Florida Department of Health. http://www.floridahealth.gov/providerand-partner-resources/fccdhh/_documents/2017-report-websitefriendly.pdf 
Garberoglio, C. L., Cawthon, S., \& Sales, A. (2017). Postsecondary achievement of deaf people in Florida: 2017. U.S. Department of Education, Office of Special Education Programs, National Deaf Center on Postsecondary Outcomes.

Graybill, P., Aggas, J., Dean, R. K., Demers, S., Finigan, E. G., \& Pollard, R. Q., Jr. (2010). A community-participatory approach to adapting survey items for Deaf individuals and American Sign Language. Field Methods.

Hall, W. C. (2017). What you don't know can hurt you: The risk of language deprivation by impairing sign language development in deaf children. Maternal and Child Health Journal, 21(5), 961-965.

Hall, W. C., Levin, L. L., \& Anderson, M. L. (2017). Language deprivation syndrome: A possible neurodevelopmental disorder with sociocultural origins. Social Psychiatry and Psychiatric Epidemiology, 52(6), 761-776.

Hawkins-Cox, D., Harris, J. R., Brownson, R. C., Ammerman, A. S., \& Gray, B. S. (2014). Prevention Research Centers Program: Researcher-community partnership for high-impact results. Encyclopedia of Primary Prevention and Health Promotion, 248-273.

Heckathorn, D. D. (2002). Respondent-driven sampling II: Deriving valid population estimates from chain-referral samples of hidden populations. Social Problems, 49(1), 11-34. https://doi.org/10.1525/sp.2002.49.1.11

Israel, B. A., Coombe, C. M., Cheezum, R. R., Schulz, A. J., McGranaghan, R. J., Lichtenstein, R., Reyes, A. G., Clement, J., \& Burris, A. (2010). Community-based participatory research: A capacity-building approach for policy advocacy aimed at eliminating health disparities. American Journal of Public Health, 100(11), 2094-2102.

Israel, B. A., Parker, E. A., Rowe, Z., Salvatore, A., Minkler, M., López, J., Butz, A., Mosley, A., Coates, L., Lambert, G., Potito, P. A., Brenner, B., Rivera, M., Romero, H., Thompson, B., Coronado, G., \& Halstead, S. (2005). Community-based participatory research: Lessons learned from the Centers for Children's Environmental Health and Disease Prevention Research. Environmental Health Perspectives, 113(10), 1463-1471. https://doi.org/10.1289/ehp.7675

Kroenke, K., Spitzer, R. L., \& Williams, J. B. W. (2003). The Patient Health Questionnaire-2: Validity of a two-item depression screener. Medical Care, 41(11), 1284-1292. JSTOR.

Kushalnagar, P., Engelman, A., \& Sadler, G. (2018). Deaf patientprovider communication and lung cancer screening: Health Information National Trends survey in American Sign Language (HINTS-ASL). Patient Education and Counseling, 0(0). https://doi.org/10.1016/j.pec.2018.03.003

Kushalnagar, P., Harris, R., Paludneviciene, R., \& Hoglind, T. (2017). Health Information National Trends survey in American Sign Language (HINTS-ASL): Protocol for the cultural adaptation and linguistic validation of a national survey. JMIR Research Protocols, 6(9), e172.

Kushalnagar, P., Ryan, C., Smith, S., \& Kushalnagar, R. (2017). Critical health literacy in American deaf college students. Health Promotion International. https://doi.org/10.1093/heapro/dax022

Lamstein, A. (2018). choroplethr: Simplify the creation of choropleth maps in R (Version 3.6.3) [R]. https://CRAN.R-project.org/package $=$ choroplethr
Logan, B. N., Davis, L., \& Parker, V. G. (2010). An interinstitutional academic collaborative partnership to end health disparities. Health Education \& Behavior, 37(4), 580-592. https://doi.org/10.1177/1090198110363378

McKee, M. M., Barnett, S. L., Block, R. C., \& Pearson, T. A. (2011). Impact of communication on preventive services among deaf American Sign Language users. American Journal of Preventive Medicine, 41(1), 75-79.

McKee, M. M., Paasche-Orlow, M. K., Winters, P. C., Fiscella, K., Zazove, P., Sen, A., \& Pearson, T. (2015). Assessing health literacy in Deaf American Sign Language users. Journal of Health Communication, 20 Suppl 2, 92-100. https://doi.org/10.1080/10810730.2015.1066468

McKee, M. M., Schlehofer, D., \& Thew, D. (2013). Ethical issues in conducting research with Deaf populations. American Journal of Public Health, 103(12), 2174-2178. https://doi.org/10.2105/AJPH.2013.301343

McKee, M. M., Thew, D., Starr, M., Kushalnagar, P., Reid, J. T., Graybill, P., Velasquez, J., \& Pearson, T. (2012). Engaging the deaf American Sign Language community: Lessons from a community-based participatory research center. Progress in Community Health Partnerships: Research, Education, and Action, 6(3), 321.

Mikesell, L., Bromley, E., \& Khodyakov, D. (2013). Ethical community-engaged research: A literature review. American Journal of Public Health, 103(12), e7-e14. https://doi.org/10.2105/AJPH.2013.301605

Minkler, M. (2005). Community-based research partnerships: Challenges and opportunities. Journal of Urban Health: Bulletin of the New York Academy of Medicine, 82(Suppl 2), ii3ii12. https://doi.org/10.1093/jurban/jti034

Moore, C. G., Carter, R. E., Nietert, P. J., \& Stewart, P. W. (2011). Recommendations for planning pilot studies in clinical and translational research. Clinical and Translational Science, 4(5), 332-337. https://doi.org/10.1111/j.17528062.2011.00347.x

Pertz, L., Plegue, M., Diehl, K., Zazove, P., \& McKee, M. (2018). Addressing mental health needs for Deaf patients through an intergrated health care model. The Journal of Deaf Studies and Deaf Education, 23(3), 240-248. https://doi.org/10.1093/deafed/eny002

Pollard, R. Q., Jr. (1998). Psychopathology. In M. Marschark \& M. D. Clark (Eds.), Psychological perspectives on deafness (Vol. 2, pp. 171-197). Lawrence Erlbaum Associates.

Simonds, V. W., Wallerstein, N., Duran, B., \& Villegas, M. (2013). Community-based participatory research: Its role in future cancer research and public health practice. Preventing Chronic Disease, 10. https://doi.org/10.5888/pcd10.120205

Wiernik, B. M. (2019, October 11). Preprint templates. https://doi.org/10.17605/OSF.IO/HSV6A

Wethington, E., Breckman, R., Meador, R., Reid, M. C., Sabir, M., Lachs, M., \& Pillemer, K. A. (2007). The CITRA pilot studies program: Mentoring translational research. The Gerontologist, 47(6), 845-850.

Zazove, P., Meador, H. E., Reed, B. D., \& Gorenflo, D. W. (2013). Deaf persons' english reading levels and associations with epidemiological, educational, and cultural factors. Journal of Health Communication, 18(7), 760-772. https://doi.org/10.1080/10810730.2012.743633

This preprint was formatted using Scholarly Preprint Word Templates created by Brenton M. Wiernik. For more information: https://osf.io/hsv6a/. 